International Journal of Pure and Applied Mathematics

Volume 111 No. 2 2016, 233-248

ISSN: 1311-8080 (printed version); ISSN: 1314-3395 (on-line version)

url: http://www.ijpam.eu

doi: 10.12732 /ijpam.v111i2.9

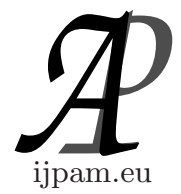

\title{
SIMPLE GRAPHOIDAL COVERING NUMBER OF PRODUCT OF GRAPHS
}

\author{
G. Venkat Narayanan ${ }^{1 \S}$, J. Suresh Suseela ${ }^{2}$, R. Kala ${ }^{3}$ \\ ${ }^{1}$ Department of Mathematics \\ St. Joseph's College of Engineering \\ Chennai, INDIA \\ ${ }^{2}$ Department of Mathematics \\ St. John's College \\ Tirunelveli, INDIA \\ ${ }^{3}$ Department of Mathematics \\ Manonmaniam Sundaranar University \\ Tirunelveli, INDIA
}

\begin{abstract}
A graphoidal cover of $\mathrm{G}$ is a set $\psi$ of (not necessarily open) paths in $\mathrm{G}$, such that every path in $\psi$ has at least two vertices, every vertex of $\mathrm{G}$ is an internal vertex of at most one path in $\psi$ and every edge of $\mathrm{G}$ is in exactly one path in $\psi$. The minimum cardinality of a graphoidal cover of $\mathrm{G}$ is called the graphoidal covering number of $\mathrm{G}$ and is denoted by $\eta$. If every two paths in $\psi$ have at most one common vertex, then it is called simple graphoidal cover of $\mathrm{G}$. The minimum cardinality of a simple graphoidal cover of $\mathrm{G}$ is called simple graphoidal covering number of $\mathrm{G}$ and is denoted by $\eta_{s}$. Here we determine the simple graphoidal covering number of Product of Graphs.
\end{abstract}

AMS Subject Classification: $05 \mathrm{C} 70,05 \mathrm{C} 76$

Key Words: simple graphoidal covering number, Cartesian product, strong product, lexicographic product

Received: $\quad$ August 24, 2016

Revised: $\quad$ November 3, 2016

Published: December 11, 2016

${ }^{\S}$ Correspondence author (c) 2016 Academic Publications, Ltd. url: www.acadpubl.eu 


\section{Introduction}

By a graph $\mathrm{G}=(\mathrm{V}, \mathrm{E})$, we mean a finite undirected graph without loop or multiple edges. The order and size of the $\mathrm{G}$ are denoted by $\mathrm{p}$ and $\mathrm{q}$ respectively. For theoretical terminology of graph we refer Harary [10]. All the graphs considered in this paper are assumed to be connected and nontrivial. If $P=\left(v_{1}, v_{2}, v_{3}, \ldots v_{n}\right)$ be a path or cycle in a graph $\mathrm{G}$, the vertices $v_{2}, v_{3}, v_{4}, \ldots . v_{n-1}$ are called internal vertices of $\mathrm{P}$ and $v_{1}, v_{n}$ are called external vertices of $\mathrm{P}$. Two paths $\mathrm{P}$ and $\mathrm{Q}$ are said to be internally disjoint if no vertex of $\mathrm{G}$ is an internal vertex of both $\mathrm{P}$ and $\mathrm{Q}$. The concept of graphoidal cover was introduced by Acharya and Sampath Kumar [1][2]. Arumugam and Pakkiam $[5],[6],[7],[8],[9]$ determined the graphoidal covering number of several families of curve. Further Arumugam and Suresh Suseela [3] introduced acyclic graphoidal cover. The concept of simple graphoidal cover was introduced by Arumugam and Shahul Hamid [4].

Definition 1. (see [1]) A graphoidal cover of $\mathrm{G}$ is a set $\psi$ of (not necessarily open) paths in $\mathrm{G}$ satisfying the following conditions.

(i) Every path in $\psi$ has at least two vertices.

(ii) Every vertex of $\mathrm{G}$ is an internal vertex of at most one path in $\psi$.

(iii) Every edge of $\mathrm{G}$ is in exactly one path in $\psi$.

The minimum cardinality of a graphoidal cover of $\mathrm{G}$ is called the graphoidal covering number of $\mathrm{G}$ and is denoted by $\eta$.

Definition 2. (see [4]) A simple graphoidal cover of a graph $\mathrm{G}$ is a graphoidal cover $\psi$ of $\mathrm{G}$ such that any two paths in $\psi$ have at most one vertex in common. The minimum cardinality of a simple graphoidal cover of $\mathrm{G}$ is called simple graphoidal covering number of $\mathrm{G}$ and is denoted by $\eta_{s}$.

Definition 3. (see [4]) Let $\psi$ be a collection of internally disjoint paths in $\mathrm{G}$. A vertex of $\mathrm{G}$ is said to be an interior vertex of if it is an internal vertex of some path in $\psi$. Any vertex which is not an interior vertex of is said to be an exterior vertex of $\psi$.

Theorem 4. For any simple graphoidal cover $\psi$ of a $(p, q)$ of graph $G$, let $t_{\psi}$ denote the number of exterior vertices of $\psi$ and let $t=\min t_{\psi}$, where the minimum is taken overall simple graphoidal covers $\psi$ of $G$, Then $\eta_{s}(G)=q-p+t$.

Corollary 5. For any graph $G, \eta_{s}(G) \geq q-p$. Moreover, the following are equivalent 
(i) $\eta_{s}(G)=q-p$.

(ii) There exists a simple graphoidal cover of $G$ without exterior vertices

(iii) There exists a set $P$ of internally disjoint and edge disjoint paths without exterior vertices such that any two paths in $P$ have at most one vertex in common.

Theorem 6. For the complete graph $K_{n}(n \geq 3)$, we have

$$
\eta_{s}\left(K_{n}\right)= \begin{cases}\frac{(n-1)(n-2)}{2} & \text { if } n=4 \\ \frac{n(n-3)}{2} & \text { if } n \text { is odd } \\ \text { if } n>4 \text { and } n \text { is even }\end{cases}
$$

Given two graphs $\mathrm{G}$ and $\mathrm{H}$, their product is a graph whose vertex set is $\mathrm{V}(\mathrm{G})$ $\mathrm{X} \mathrm{V}(\mathrm{H})=\{(g, h): g \in V(G)$ and $h \in V(H)\}$, while the edge set varies according to the nature of the product. Various kinds of graph products are defined in [12]. We consider only Cartesian product, Strong product and Lexicographic product of two paths or cycles.

The Cartesian Product of two graphs $\mathrm{G}$ and $\mathrm{H}$ denoted by $\mathrm{G} \circ \mathrm{H}$ has the edge set $\mathrm{E}(\mathrm{G} \circ \mathrm{H})=\left\{\left((g, h),\left(g^{\prime}, h^{\prime}\right)\right): g=g^{\prime},\left(h, h^{\prime}\right) \in E(H)\right.$ or $h=h^{\prime},\left(g, g^{\prime}\right) \in$ $E(G)\}$.

The Strong Product of graphs $\mathrm{G}$ and $\mathrm{H}$ is denoted by $\mathrm{G} \otimes \mathrm{H}$ where $\mathrm{E}(\mathrm{G}$ $\otimes \mathrm{H})=\left\{\left((g, h),\left(g^{\prime}, h^{\prime}\right)\right): g=g^{\prime},\left(h, h^{\prime}\right) \in E(H)\right) ;\left(g, g^{\prime}\right) \in E(G), h=h^{\prime}$ or $\left(g, g^{\prime}\right) \in E(G)$ and $\left.\left(h, h^{\prime}\right) \in E(H)\right\}$.

The Lexicographic Product or Composition of two graphs $\mathrm{G}$ and $\mathrm{H}$ is denoted by $\mathrm{G} \odot \mathrm{H}$ where $\mathrm{E}(\mathrm{G} \odot \mathrm{H})=\left\{\left((g, h),\left(g^{\prime}, h^{\prime}\right)\right):\left(g, g^{\prime}\right) \in E(G)\right.$ or $\left.g=g^{\prime},\left(h, h^{\prime}\right) \in E(H)\right\}$. For the positive integer $\mathrm{m}$ and $\mathrm{n}$, we use the following notations

$$
\begin{aligned}
& P_{m}=\left(u_{1}, u_{2} \ldots \ldots, u_{m}\right) \\
& P_{n}=\left(u_{1}, u_{2} \ldots \ldots, u_{n}\right) \\
& C_{m}=\left(u_{1}, u_{2} \ldots \ldots, u_{m}, u_{1}\right) \\
& C_{n}=\left(u_{1}, u_{2} \ldots \ldots, u_{n}, u_{1}\right)
\end{aligned}
$$

For all the product graphs $\mathrm{G}$, we have considered the vertex set $\mathrm{V}(\mathrm{G})=\left\{w_{i j}\right.$ : $1 \leq i \leq m ; 1 \leq j \leq n\}$ where $w_{i, j}=\left(u_{i}, v_{j}\right)$. 


\section{Main Results}

Theorem 7. If $G=P_{m} \circ P_{n}$, where $m, n \geq 2$ then

$$
\eta_{s}(G)= \begin{cases}q-p+1 & \text { if } m=n=2 \text { or } m, n \geq 3 \\ q-p+2 & \text { if } m=2 \text { and } n \geq 3\end{cases}
$$

Proof. The Edge set $\mathrm{E}(\mathrm{G})=\left\{w_{i j} w_{i(j+1)}: 1 \leq i \leq m, 1 \leq j \leq(n-1)\right\} \cup$ $\left\{w_{i j} w_{(i+1) j}: 1 \leq i \leq(m-1), 1 \leq j \leq n\right\}$.

When $\mathrm{m}=2$ and $n \geq 3$, The collection of paths of $\mathrm{G}$ are

$P=\left(w_{11}, w_{12}, w_{22}, w_{21}, w_{11}\right)$

$Q=\left(w_{12}, w_{13}, \ldots \ldots w_{1 n}, w_{2 n}\right)$

$R=\left(w_{22}, w_{23}, \ldots \ldots, w_{2 n}\right)$

$S=E(G)-P \cup Q \cup R$

is a simple graphoidal cover of $\mathrm{G}$ in which $w_{11}, w_{2 n}$ are not internal. Therefore $\eta_{s}(G) \leq q-p+2$. Now, let $\psi$ be any simple graphoidal cover of G. If $\psi$ contains only paths, then atleast three vertices can not be made internal. If $\psi$ contains atleast one cycle, then atleast two vertices can not be made internal. There foret $\psi \geq 2$. Hence $\eta_{s}(G) \geq q-p+2$. Thus $\eta_{s}(G)=q-p+2$.

When $\mathrm{m}, \mathrm{n} \geq 3$, then we have the following four cases.

Case (i) when $\mathrm{m}=\mathrm{n}=$ odd, The collection of paths of $\mathrm{G}$ are

$$
\begin{aligned}
& P=\left(w_{11}, w_{12}, w_{22}, w_{21}, w_{11}\right) \\
& Q_{i}=\left(w_{2 i}, w_{2(i+1)}, w_{3(i+1)}, w_{3 i}, w_{2 i}\right): i=2,4,6 \ldots .(n-1) \\
& R_{i}=\left(w_{2 i}, w_{1 i}, w_{1(i+1)}, w_{2(i+1)}, w_{2 i}\right): i=3,5,7 \ldots .(n-2) \\
& S_{i}=\left(w_{i 2}, w_{i 1}, w_{(i+1) 1}, w_{(i+1) 2}, w_{i 2}\right): i=3,5,7 \ldots .(m-2) \\
& T_{i}=\left(w_{i j}, w_{i(j+1)}, w_{(i+1)(j+1)}, w_{(i+1) j}, w_{i j}\right): i=4,6 . .(m-1) ; j=2,4 . .(n-1) \\
& U_{i}=\left(w_{i j}, w_{i(j+1)}, w_{(i-1)(j+1)}\right): i=4,6,8 \ldots(m-1) ; j=3,5 \ldots(n-2) \\
& V=\left(w_{1(n-1)}, w_{1 n}, w_{2 n}\right) \\
& W=\left(w_{(m-1) 1}, w_{m 1}, w_{m 2}\right) \\
& X=E(G)-P \cup Q_{i} \cup R_{i} \cup S_{i} \cup T_{i} \cup U_{i} \cup V \cup W
\end{aligned}
$$

is a simple graphoidal cover of $\mathrm{G}$ in which $w_{11}$ is not internal. Therefore $\eta_{s}(G) \leq$ $q-p+1$. Now, let $\psi$ be any simple graphoidal cover of G. If $\psi$ contains only paths, then atleast three vertices can not be made internal. If $\psi$ contains atleast 
one cycle, then atleast one vertex can not be made internal. Thereforet $\psi \geq 1$. Hence $\eta_{s}(G) \geq q-p+1$. Thus $\eta_{s}(G)=q-p+1$.

Case(ii) When $\mathrm{m}=$ odd and $\mathrm{n}=$ even, The collection of paths of $\mathrm{G}$ are

$$
\begin{aligned}
& P=\left(w_{11}, w_{12}, w_{22}, w_{21}, w_{11}\right) \\
& Q_{i}=\left(w_{2 i}, w_{2(i+1)}, w_{3(i+1)}, w_{3 i}, w_{2 i}\right): i=2,4,6 \ldots .(n-2) \\
& R_{i}=\left(w_{2 i}, w_{1 i}, w_{1(i+1)}, w_{2(i+1)}, w_{2 i}\right): i=3,5,7 \ldots .(n-1) \\
& S_{i}=\left(w_{i 2}, w_{i 1}, w_{(i+1) 1}, w_{(i+1) 2}, w_{i 2}\right): i=3,5,7 \ldots(m-2) \\
& T_{i}=\left(w_{i(n-1)}, w_{i n}, w_{(i+1) n}, w_{(i+1)(n-1)}, w_{i(n-1)}\right): i=3,5, \ldots(m-2) \\
& U_{i}=\left(w_{i 2}, w_{i 3}, w_{(i+1) 3}, w_{(i+1) 2}, w_{i 2}\right): i=4,6, \ldots . .(m-1) \\
& V_{i}=\left(w_{i(n-1)}, w_{i(n-2)}, w_{(i+1)(n-2)}, w_{(i+1)(n-1)}, w_{i(n-1)}\right): i=4,6 . .(m-1) \\
& W_{i}=\left(w_{i j}, w_{i(j+1)}, w_{(i+1)(j+1)}, w_{(i+1) j}, w_{i j}\right): i=4,6, . .(m-1), j=4,6, \ldots(n-4) \\
& X_{i}=\left(w_{i j}, w_{i(j+1)}, w_{(i-1) j}\right): i=4,6, \ldots(m-1), j=3,5 \ldots(n-5) \\
& Y=\left(w_{(m-1) 1}, w_{m 1}, w_{m 2}\right) \\
& Z=\left(w_{m(n-1)}, w_{m n}, w_{(m-1) n}\right) \\
& Z^{\prime}=E(G)-P \cup Q_{i} \cup R_{i} \cup S_{i} \cup T_{i} \cup U_{i} \cup V_{i} \cup W_{i} \cup X_{i} \cup Y \cup Z
\end{aligned}
$$

is a simple graphoidal cover of $\mathrm{G}$ in which $w_{11}$ is not internal. Therefore $\eta_{s}(G) \leq$ $q-p+1$. Now, let $\psi$ be any simple graphoidal cover of $\mathrm{G}$. If $\psi$ contains only paths, then atleast three vertices can not be made internal. If $\psi$ contains atleast one cycle, then atleast one vertex can not be made internal. Thereforet $\psi \geq 1$. Hence $\eta_{s}(G) \geq q-p+1$. Thus $\eta_{s}(G)=q-p+1$.

Case (iii) When $\mathrm{m}=$ even and $\mathrm{n}=$ odd, The collection of paths of $\mathrm{G}$ are

$$
\begin{aligned}
& P=\left(w_{11}, w_{12}, w_{22}, w_{21}, w_{11}\right) \\
& Q_{i}=\left(w_{2 i}, w_{2(i+1)}, w_{3(i+1)}, w_{3 i}, w_{2 i}\right): i=2,4,6 \ldots(n-1) \\
& R_{i}=\left(w_{2 i}, w_{1 i}, w_{1(i+1)}, w_{2(i+1)}, w_{2 i}\right): i=3,5,7 \ldots(n-2) \\
& S_{i}=\left(w_{i 2}, w_{i 1}, w_{(i+1) 1}, w_{(i+1) 2}, w_{i 2}\right): i=3,5,7 \ldots .(m-1) \\
& T_{i}=\left(w_{i j}, w_{i(j+1)}, w_{(i+1)(j+1)}, w_{(i+1) j}, w_{i j}\right): i=4,6, . .(m-2), j=2,4, . .(n-1) \\
& U_{i}=\left(w_{m i}, w_{m(i+1)}, w_{(m-1)(i+1)}\right): i=2,3, \ldots .(n-1) \\
& V_{i}=\left(w_{i j}, w_{i(j+1)}, w_{(i-1) j}\right): i=4,6, \ldots(m-2), j=3,5, \ldots(n-2) \\
& W=\left(w_{1(n-1)}, w_{1 n}, w_{2 n}\right) \\
& X=E(G)-P \cup Q_{i} \cup R_{i} \cup S_{i} \cup T_{i} \cup U_{i} \cup V_{i} \cup W
\end{aligned}
$$


is a simple graphoidal cover of $\mathrm{G}$ in which $w_{11}$ is not internal. Therefore $\eta_{s}(G) \leq$ $q-p+1$. Now, let $\psi$ be any simple graphoidal cover of $\mathrm{G}$. If $\psi$ contains only paths, then atleast three vertices can not be made internal. If $\psi$ contains atleast one cycle, then atleast one vertex can not be made internal. Thereforet $\psi \geq 1$. Hence $\eta_{s}(G) \geq q-p+1$. Thus $\eta_{s}(G)=q-p+1$.

Case(iv) When $\mathrm{m}=\mathrm{n}=$ even or $\mathrm{m}=\mathrm{n}=2$. The collection of paths of $\mathrm{G}$ are

$$
\begin{aligned}
& P=\left(w_{11}, w_{12}, w_{22}, w_{21}, w_{11}\right) \\
& Q_{i}=\left(w_{2 i}, w_{2(i+1)}, w_{3(i+1)}, w_{3 i}, w_{2 i}\right): i=2,4, \ldots .(n-2) \\
& R_{i}=\left(w_{2 i}, w_{1 i}, w_{1(i+1)}, w_{2(i+1)}, w_{2 i}\right): i=3,5, \ldots .(n-1) \\
& S_{i}=\left(w_{i 2}, w_{i 1}, w_{(i+1) 1}, w_{(i+1) 2}, w_{i 2}\right): i=3,5, \ldots(m-1) \\
& T_{i}=\left(w_{i(n-1)}, w_{i n}, w_{(i+1) n}, w_{(i+1)(n-1)}, w_{i(n-1)}\right): i=3,5, \ldots(m-1) \\
& U_{i}=\left(w_{i 2}, w_{i 3}, w_{(i+1) 3}, w_{(i+1) 2}, w_{i 2}\right): i=4,6,8 \ldots(m-2) \\
& V_{i}=\left(w_{i(n-1)}, w_{i(n-2)}, w_{(i+1)(n-2)}, w_{(i+1)(n-1)}, w_{i(n-1)}\right): i=4,6, \ldots(m-2) \\
& W_{i}=\left(w_{i j}, w_{i(j+1)}, w_{(i+1)(j+1)}, w_{(i+1) j}, w_{i j}\right): i=4,6, \ldots(m-2), j=4,6, \ldots(n-4) \\
& Y_{i}=\left(w_{i j}, w_{i(j+1)}, w_{(i-1)(j+1)}\right): i=4,6, \ldots(m-2), j=3,5, \ldots(n-5) \\
& Z_{i}=\left(w_{m i}, w_{m(i+1)}, w_{(m-1)(i+1)}\right): i=2,3 . .(n-3) \\
& \quad Z=E(G)-P \cup Q_{i} \cup R_{i} \cup S_{i} \cup T_{i} \cup U_{i} \cup V_{i} \cup W_{i} \cup Y_{i} \cup Z_{i}
\end{aligned}
$$

is a simple graphoidal cover of $\mathrm{G}$ in which $w_{11}$ is the only vertex which is not internal. Therefore $\eta_{s}(G) \leq q-p+1$. Now, let $\psi$ be any simple graphoidal cover of $\mathrm{G}$. If $\psi$ contains only paths, then atleast three vertices can not be made internal. If $\psi$ contains atleast one cycle, then atleast one vertex can not be made internal. Thereforet $\psi \geq 1$. Hence $\eta_{s}(G) \geq q-p+1$. Thus $\eta_{s}(G)=q-p+1$.

Theorem 8. If $G=C_{m} \circ C_{n}$, then $\eta_{s}(G)=q-p+1$.

Proof. The edge set $\mathrm{E}(\mathrm{G})=\left\{w_{i j} w_{i(j+1)}: 1 \leq i \leq m, 1 \leq j \leq(n-1)\right\} \cup$ $\left\{w_{i n} w_{i 1}: 1 \leq i \leq m\right\} \cup\left\{w_{i j} w_{(i+1) j}: 1 \leq i \leq(m-1), 1 \leq j \leq n\right\} \cup\left\{w_{m j} w_{1 j}:\right.$ $1 \leq j \leq n\}$. The collection of paths of $\mathrm{G}$ are

$$
\begin{aligned}
& P_{i}=\left(w_{i 1}, w_{i 2}, w_{i 3}, \ldots w_{i n}, w_{i 1}\right), 1 \leq i \leq m \\
& Q=\left(w_{11}, w_{21}, \ldots, w_{m 1}, w_{11}\right) \\
& R=E(G)-P_{i} \cup Q
\end{aligned}
$$


is a simple graphoidal cover of $\mathrm{G}$ in which $w_{11}$ is not internal. Therefore $\eta_{s}(G) \leq$ $q-p+1$. Now, let $\psi$ be any simple graphoidal cover of G. Here we have two cases.

Case(i) when $\mathrm{m}=\mathrm{n}$, If $\psi$ contains only paths, then atleast $\mathrm{mn}-(\mathrm{m}+\mathrm{n})>$ 1 vertices can not be made internal, since every path is of length 2 . If $\psi$ contains atleast one cycle, then atleast one vertex can not be made internal. Thereforet $\psi \geq 1$. Hence $\eta_{s}(G) \geq q-p+1$. Thus $\eta_{s}(G)=q-p+1$.

Case(ii) when $\mathrm{m} \neq \mathrm{n}$, If $\psi$ contains only paths, then atleast $\mathrm{mn}-(\mathrm{m}+\mathrm{n})$ 1 vertices can not be made internal, since every path is of length 2 . If $\psi$ contains atleast one cycle,then atleast one vertex can not be made internal. Thereforet $\psi 1$. Hence $\eta_{s}(G) \geq q-p+1$. Thus $\eta_{s}(G)=q-p+1$.

Theorem 9. If $G=P_{m} \circ C_{n}$ where $m \geq 2, n \geq 3$, then $\eta_{s}(G)=q-p+2$

Proof. The edge set $\mathrm{E}(\mathrm{G})=\left\{w_{i j} w_{i(j+1)}: 1 \leq i \leq m, 1 \leq j \leq(n-1)\right\} \cup$ $\left\{w_{i n} w_{i 1}: 1 \leq i \leq m\right\} \cup\left\{w_{i j} w_{(i+1) j}: 1 \leq i \leq(m-1), 1 \leq j \leq n\right\}$. The collection of paths of $\mathrm{G}$ are

$P_{i}=\left(w_{i 1}, w_{i 2}, w_{i 3}, \ldots, w_{i n}, w_{i 1}\right), 1 \leq i \leq m$.

$Q=\left(w_{11}, w_{21}, \ldots, w_{m 1}, w_{11}\right)$

$R=E(G)-P_{i} \cup Q$

is a simple graphoidal cover of $\mathrm{G}$ in which $w_{11}$ and $w_{n 1}$ are not internal. Therefore $\eta_{s}(G) \leq q-p+2$. Now, let $\psi$ be any simple graphoidal cover of G. If $\psi$ can contains only paths, then atleast four vertices can not be made internal. If $\psi$ can contains atleast one cycle, then atleast two vertices can not be made internal. Thereforet $\psi \geq 2$. Hence $\eta_{s}(G) \geq q-p+2$. Thus $\eta_{s}(G)=q-p+2$.

Theorem 10. For the graph $G=P_{m} \odot P_{n}$, where $m, n \geq 2$ then

$$
\eta_{s}(G)= \begin{cases}4 & \text { if } m=n=2 \\ q-p+1 & \text { if } m=2 \text { and } n=3 \text { or } m=n=3 \\ q-p & \text { if } m \geq 3 \text { and } n \geq 4 \text { or } m=2 \text { and } n \geq 4\end{cases}
$$

Proof. The edge set $\mathrm{E}(\mathrm{G})=\left\{w_{i j} w_{(i+1) k}: 1 \leq i \leq(m-1), i \leq j \leq n, 1 \leq\right.$ $k \leq n\} \cup\left\{w_{i j} w_{i(j+1)}: 1 \leq i \leq m, 1 \leq j \leq(n-1)\right\}$.

When $\mathrm{m}=\mathrm{n}=2$, Then $\mathrm{G}=K_{4}$, by Theorem $6, \eta_{s}(G)=4$

When $\mathrm{m}=2$ and $\mathrm{n}=3$, the collection of paths of $\mathrm{G}$ are $P_{i}=\left(w_{1 i}, w_{1(i+1)}, w_{2 i}, w_{1 i}\right), 1 \leq i \leq n-1$ 
$Q=\left(w_{11}, w_{2 n}, w_{1 n}\right)$

$R=E(G)-P_{i} \cup Q$

is a simple graphoidal graph in which $w_{11}$ is not internal. Therefore $\eta_{s}(G) \leq$ $q-p+1$. Now let $\psi$ be any graphoidal cover of $\mathrm{G}$. If $\psi$ contains only paths, then atleast four vertices can not be made internal. If $\psi$ contains atleast one cycle, then atleast one vertex can not be made internal. Thereforet $\psi \geq 1$. Hence $\eta_{s}(G) \geq q-p+1$. Thus $\eta_{s}(G)=q-p+1$.

when $\mathrm{m}=2$ and $\mathrm{n} \geq 4$, the collection of path of $\mathrm{G}$ are

$P_{i}=\left(w_{1 i}, w_{1(i+1)}, w_{2 i}, w_{1 i}\right), 1 \leq i \leq(n-1)$.

$Q=\left(w_{22}, w_{11}, w_{2 n}\right)$

$R=\left(w_{12}, w_{2 n}, w_{1 n}\right)$

$S=E(G)-P_{i} \cup Q \cup R$

is a simple graphoidal graph in which all vertices made internal. By corollary (5), $\eta_{s}(G)=q-p$.

When $\mathrm{m}=3$ and $\mathrm{n}=3$, the collection of paths of $\mathrm{G}$ are

$P_{i}=\left(w_{1 i}, w_{1(i+1)}, w_{2 i}, w_{1 i}\right), 1 \leq i \leq(n-1)$

$Q=\left(w_{21}, w_{31}, w_{32}, w_{21}\right)$

$R=\left(w_{22}, w_{23}, w_{33}, w_{22}\right)$

$S=E(G)-P_{i} \cup Q \cup R$

is a simple graphoidal graph in which $w_{11}$ is the only vertex not internal. Therefore $\eta_{s}(G) \leq q-p+1$. Now let $\psi$ be any graphoidal cover of $\mathrm{G}$. If $\psi$ contains only paths, then atleast four vertices can not be made internal. If $\psi$ contains atleast one cycle, then atleast one vertex can not be made internal. Thereforet $\psi \geq 1$. Hence $\eta_{s}(G) \geq q-p+1$. Thus $\eta_{s}(G)=q-p+1$.

When $m \geq 3$ and $n \geq 4$, then we have following cases.

Case (i) when $\mathrm{m}=\mathrm{n}=$ odd, the collection of paths of $\mathrm{G}$ are

$$
\begin{aligned}
& P_{i}=\left(w_{1 i}, w_{1(i+1)}, w_{2 i}, w_{1 i}\right) ; 1 \leq i \leq(n-1) \\
& Q_{i}=\left(w_{i j}, w_{(i+1) j}, w_{(i+1)(j+1)}, w_{i j}\right): i=2,3,4 \ldots(m-1), j=1,3,5 . .(n-2) \\
& R_{i}=\left(w_{i(n-1)}, w_{i n}, w_{(i+1) n}, w_{i(n-1)}\right) ; i=2,4,6 \ldots(m-1) \\
& S=\left(w_{22}, w_{11}, w_{2 n}\right) \\
& T=E(G)-P_{i} \cup Q_{i} \cup R_{i} \cup S
\end{aligned}
$$


is a simple graphoidal cover of $\mathrm{G}$ in which all the vertices made internal. By corollary $(5), \eta_{s}(G)=q-p$.

Case (ii) when $\mathrm{m}=$ odd and $\mathrm{n}=$ even, then the collection of path of $\mathrm{G}$ are

$P_{i}=\left(w_{1 i}, w_{1(i+1)}, w_{2 i}, w_{1 i}\right): 1 \leq i \leq(n-1)$

$Q_{i}=\left(w_{i j}, w_{(i+1) j}, w_{(i+1)(j+1)}, w_{i j}\right): i=2,3,4 \ldots(m-1), j=1,3,5 \ldots(n-1)$

$R=\left(w_{1 n}, w_{2 n}, w_{3 n}\right)$

$S=\left(w_{22}, w_{11}, w_{2 n}\right)$

$T=E(G)-P_{i} \cup Q_{i} \cup R \cup S$

is a simple graphoidal cover of $\mathrm{G}$ in which all the vertices made internal. By corollary $(5), \eta_{s}(G)=q-p$.

Case (iii) when $\mathrm{m}=$ even and $\mathrm{n}=$ odd, then the collection of path of $\mathrm{G}$ are

$P_{i}=\left(w_{1 i}, w_{1(i+1)}, w_{2 i}, w_{1 i}\right): 1 \leq i \leq(n-1)$

$Q_{i}=\left(w_{i j}, w_{(i+1) j}, w_{(i+1)(j+1)}, w_{i j}\right): i=2,3,4 \ldots(m-1), j=1,3,5 \ldots(n-2)$

$R_{i}=\left(w_{i(n-1)}, w_{i n}, w_{(i+1) n}, w_{i(n-1)}\right): i=3,5,7, \ldots(m-1)$

$S=\left(w_{1 n}, w_{2 n}, w_{3 n}\right)$

$T=\left(w_{22}, w_{11}, w_{2 n}\right)$

$U=E(G)-P_{i} \cup Q_{i} \cup R_{i} \cup S \cup T$

is a simple graphoidal cover of $\mathrm{G}$ in which all the vertices made internal. By Corollary $(5), \eta_{s}(G)=q-p$.

Case (iv) when $\mathrm{m}=\mathrm{n}=$ even,then the collection of path of $\mathrm{G}$ are

$P_{i}=\left(w_{1 i}, w_{1(i+1)}, w_{2 i}, w_{1 i}\right), 1 \leq i \leq(n-1)$

$Q_{i}=\left(w_{i j}, w_{(i+1) j}, w_{(i+1)(j+1)}, w_{i j}\right): i=2,3,4 \ldots(m-1), j=1,3,5 \ldots(n-1)$

$R=\left(w_{1 n}, w_{2 n}, w_{3 n}\right)$

$S=\left(w_{22}, w_{11}, w_{2 n}\right)$

$T=E(G)-P_{i} \cup Q_{i} \cup R \cup S$

is a simple graphoidal cover of $\mathrm{G}$ in which all the vertices made internal. By corollary $(5)), \eta_{s}(G)=q-p$.

Theorem 11. For the graph $G=C_{m} \odot C_{n}$, where $m, n \geq 3$, then

$$
\eta_{s}(G)= \begin{cases}q-p+1 & \text { if } m=3 \text { and } n \geq 3 \\ q-p & \text { if } m=n \geq 4\end{cases}
$$


Proof. Let $\mathrm{E}(\mathrm{G})=\left\{w_{i j} w_{(i+1) k}: 1 \leq i \leq(n-1) ; 1 \leq j \leq n ; 1 \leq k \leq\right.$ $n\} \cup\left\{w_{i j} w_{i(j+1)}: 1 \leq i \leq m ; 1 \leq j \leq(n-1)\right\} \cup\left\{w_{i 1} w_{i n}: 1 \leq i \leq m\right\}$ $\cup\left\{w_{1 j} w_{m k}: 1 \leq j \leq n ; 1 \leq k \leq n\right\}$

When $\mathrm{m}=3$ and $\mathrm{n} \geq 3$, the collection of paths of $\mathrm{G}$ are

$P_{i}=\left(w_{i 1}, w_{i 2}, w_{i 3} \ldots w_{i n}, w_{i 1}\right): 1 \leq i \leq 3$

$Q=\left(w_{11}, w_{21}, w_{31}, w_{11}\right)$

$R=E(G)-P_{i} \cup Q$

is a simple graphoidal cover of $\mathrm{G}$ in which $w_{11}$ is not internal. Therefore $\eta_{s}(G) \leq q-p+1$. Now let $\psi$ be any graphoidal cover of $\mathrm{G}$. If $\psi$ contains only paths, then no vertices can be made internal,since every path is an edge. If $\psi$ contains atleast one cycle, then atleast one vertex can not be made internal. Thereforet $\psi \geq 1$. Hence $\eta_{s}(G) \geq q-p+1$. Thus $\eta_{s}(G)=q-p+1$.

When $m \geq 4$ and $n \geq 4$, the collection of paths of $\mathrm{G}$ are

$P_{i}=\left(w_{i 1}, w_{i 2}, w_{i 3} \ldots w_{i n}, w_{i 1}\right), 1 \leq i \leq m$

$Q=\left(w_{11}, w_{21}, w_{31}, \ldots w_{m 1}, w_{11}\right)$

$R=\left(w_{22}, w_{11}, w_{2 n}\right)$

$S=E(G)-P_{i} \cup Q \cup R$

is a simple graphoidal cover of $\mathrm{G}$ in which all vertices becomes internal. By corollary $(5), \eta_{s}(G)=q-p$.

Theorem 12. For the graph $G=P_{m} \odot C_{n}$, where $m \geq 2, n \geq 3$, then

$$
\eta_{s}(G)= \begin{cases}q-p+2 & \text { if } m=2 \text { and } n=3 \\ q-p+1 & \text { if } m=n=3 \\ q-p & \text { if } m=2 \text { and } n \geq 4 \text { or } m \geq 3 \text { and } n \geq 4\end{cases}
$$

Proof. Let $\mathrm{E}(\mathrm{G})=\left\{w_{i j} w_{(i+1) k}: 1 \leq i \leq(m-1), 1 \leq j \leq n, 1 \leq k \leq\right.$ $n\} \cup\left\{w_{i j} w_{i(j+1)}: 1 \leq i \leq n, 1 \leq j \leq(n-1)\right\} \cup\left\{w_{i 1} w_{i n}: 1 \leq i \leq m\right\}$.

When $\mathrm{m}=2$ and $\mathrm{n}=3$, then the collection of paths of $\mathrm{G}$ are

$P_{i}=\left(w_{1 i}, w_{1(i+1)}, w_{2 i}, w_{1 i}\right): 1 \leq i \leq n-1$

$Q=E(G)-P_{i}$

is a simple graphoidal cover of $\mathrm{G}$ in which $w_{11}$ and $w_{2 n}$ are not internal. Therefore $\eta_{s}(G) \leq q-p+2$. Now let $\psi$ be any graphoidal cover of $\mathrm{G}$. If $\psi$ contains only paths, then no vertices can be made internal, since every path is an edge. If $\psi$ 
contains atleast one cycle, then atleast two vertices can not be made internal. Thereforet $\psi \geq 2$. Hence $\eta_{s}(G) \geq q-p+2$. Thus $\eta_{s}(G)=q-p+2$.

when $\mathrm{m}=\mathrm{n}=3$, The collection of paths of $\mathrm{G}$ are

$$
\begin{aligned}
& P_{i}=\left(w_{1 i}, w_{1(i+1)}, w_{2 i}, w_{1 i}\right): 1 \leq i \leq n-1 \\
& Q=\left(w_{21}, w_{31}, w_{32}, w_{21}\right) \\
& R=\left(w_{22}, w_{23}, w_{33}, w_{22}\right) \\
& S=E(G)-P_{i} \cup Q \cup R
\end{aligned}
$$

is a graphoidal cover of $\mathrm{G}$ in which $w_{11}$ is not internal. Therefore $\eta_{s}(G) \leq$ $q-p+1$. Now let $\psi$ be any graphoidal cover of $\mathrm{G}$. If $\psi$ contains only paths, then atleast three vertices can not be made internal. If $\psi$ contains atleast one cycle, then atleast one vertex can not be made internal. Thereforet $\psi \geq 1$. Hence $\eta_{s}(G) \geq q-p+1$. Thus $\eta_{s}(G)=q-p+1$.

when $\mathrm{m}=2$ and $n \geq 4$, The collection of paths of are

$P_{i}=\left(w_{1 i}, w_{1(i+1)}, w_{2 i}, w_{1 i}\right): 1 \leq i \leq(n-1)$

$Q=\left(w_{12}, w_{2 n}, w_{1 n}\right)$

$R=\left(w_{22}, w_{11}, w_{2 n}\right)$

$S=E(G)-P_{i} \cup Q \cup R$

is a graphoidal cover of $\mathrm{G}$ in which all vertices made internal. By corollary $(5), \eta_{s}(G)=q-p$.

When $m \geq 3$ and $n \geq 4$, then we have following cases. Case (i) when $\mathrm{m}=\mathrm{n}=$ odd, the collection of paths of $\mathrm{G}$ are

$$
\begin{aligned}
& P_{i}=\left(w_{1 i}, w_{1(i+1)}, w_{2 i}, w_{1 i}\right) ; 1 \leq i \leq n-1 \\
& Q_{i}=\left(w_{i j}, w_{(i+1) j}, w_{(i+1)(j+1)}, w_{i j}\right): i=2,3,4 \ldots(m-1), j=1,3,5 . .(n-2) \\
& R_{i}=\left(w_{i(n-1)}, w_{i n}, w_{(i+1) n}, w_{i(n-1)}\right) ; i=2,4,6 \ldots(m-1) \\
& S=\left(w_{22}, w_{11}, w_{2 n}\right) \\
& T=E(G)-P_{i} \cup Q_{i} \cup R_{i} \cup S
\end{aligned}
$$

is a simple graphoidal cover of $\mathrm{G}$ in which all the vertices made internal. By corollary $(5), \eta_{s}(G)=q-p$.

Case (ii) when $\mathrm{m}=$ odd and $\mathrm{n}=$ even, then the collection of path of $\mathrm{G}$ are

$$
\begin{aligned}
& P_{i}=\left(w_{1 i}, w_{1(i+1)}, w_{2 i}, w_{1 i}\right): 1 \leq i \leq(n-1) \\
& Q_{i}=\left(w_{i j}, w_{(i+1) j}, w_{(i+1)(j+1)}, w_{i j}\right): i=2,3,4 \ldots(m-1), j=1,3,5 \ldots(n-1)
\end{aligned}
$$


$R=\left(w_{1 n}, w_{2 n}, w_{3 n}\right)$

$S=\left(w_{22}, w_{11}, w_{2 n}\right)$

$T=E(G)-P_{i} \cup Q_{i} \cup R \cup S$

is a simple graphoidal cover of $\mathrm{G}$ in which all the vertices made internal. By corollary $(5), \eta_{s}(G)=q-p$.

Case (iii) when $\mathrm{m}=$ even and $\mathrm{n}=$ odd, then the collection of path of $\mathrm{G}$ are

$P_{i}=\left(w_{1 i}, w_{1(i+1)}, w_{2 i}, w_{1 i}\right): 1 \leq i \leq(n-1)$

$Q_{i}=\left(w_{i j}, w_{(i+1) j}, w_{(i+1)(j+1)}, w_{i j}\right): i=2,3,4 \ldots(m-1), j=1,3,5 \ldots(n-2)$

$R_{i}=\left(w_{i(n-1)}, w_{i n}, w_{(i+1) n}, w_{i(n-1)}\right): i=3,5,7, \ldots(m-1)$

$S=\left(w_{1 n}, w_{2 n}, w_{3 n}\right)$

$T=\left(w_{22}, w_{11}, w_{2 n}\right)$

$U=E(G)-P_{i} \cup Q_{i} \cup R_{i} \cup S \cup T$

is a simple graphoidal cover of $\mathrm{G}$ in which all the vertices made internal. By Corollary $(5), \eta_{s}(G)=q-p$.

Case (iv) when $\mathrm{m}=\mathrm{n}=$ even, then the collection of path of $\mathrm{G}$ are

$P_{i}=\left(w_{1 i}, w_{1(i+1)}, w_{2 i}, w_{1 i}\right), 1 \leq i \leq(n-1)$

$Q_{i}=\left(w_{i j}, w_{(i+1) j}, w_{(i+1)(j+1)}, w_{i j}\right): i=2,3,4 \ldots(m-1), j=1,3,5 \ldots(n-1)$

$R=\left(w_{1 n}, w_{2 n}, w_{3 n}\right)$

$S=\left(w_{22}, w_{11}, w_{2 n}\right)$

$T=E(G)-P_{i} \cup Q_{i} \cup R \cup S$

is a simple graphoidal cover of $\mathrm{G}$ in which all the vertices made internal. By corollary $(5)), \eta_{s}(G)=q-p$.

Theorem 13. For the graph $G=P_{m} \otimes P_{n}$, where $m, n \geq 2$ then

$$
\eta_{s}(G)= \begin{cases}q-p+2 & \text { if } m=2 \text { and } n \geq 2 \\ q-p+1 & \text { if } m=3 \text { and } n \geq 3\end{cases}
$$

Proof. Let $\mathrm{E}(\mathrm{G})=\left\{w_{i j} w_{i(j+1)}: 1 \leq i \leq m ; 1 \leq j \leq(n-1)\right\} \cup\left\{w_{i j} w_{(i+1) j}\right.$ : $1 \leq i \leq(m-1) ; 1 \leq j \leq n\} \cup\left\{w_{i j} w_{(i+1)(j+1)}: 1 \leq i \leq(m-1) ; 1 \leq j \leq\right.$ $(n-1)\} \cup\left\{w_{i j} w_{(i+1)(j-1)}: 1 \leq i \leq(m-1) ; 2 \leq j \leq n\right\}$

When $\mathrm{m}=2$ and $\mathrm{n} \geq 2$, The collection of paths of $\mathrm{G}$ are

$P_{i}=\left(w_{1 j}, w_{1(j+1)}, w_{2 j}, w_{1 j}\right): 1 \leq j \leq(n-1)$ 
$Q=E(G)-P_{i}$

is a simple graphoidal cover of $\mathrm{G}$ in which $w_{11}$ and $w_{2 n}$ are not internal. Therefore $\eta_{s}(G) \leq q-p+2$. Now let $\psi$ be any graphoidal cover of $\mathrm{G}$. If $\psi$ contains only paths, then atleast four vertices can not be made internal. If $\psi$ contains atleast one cycle, then atleast two vertices can not be made internal. Thereforet $\psi 2$. Hence $\eta_{s}(G) \geq q-p+2$. Thus $\eta_{s}(G)=q-p+2$.

When $m \geq 3$ and $n \geq 3$, Here we have following cases

Case (i) When $\mathrm{m}=\mathrm{n}=$ odd, The collection of paths of $\mathrm{G}$ are

$P_{i}=\left(w_{1 j}, w_{1(j+1)}, w_{2 j}, w_{1 j}\right): 1 \leq j \leq(n-1)$

$Q_{i}=\left(w_{i j}, w_{(i+1) j}, w_{(i+1)(j+1)}, w_{i j}\right): i=2,3 \ldots(m-1) ; j=1,3,5 \ldots(n-2)$

$R_{i}=\left(w_{i(n-1)}, w_{i n}, w_{(i+1) n}, w_{i(n-1)}\right): i=2,4 \ldots(m-1)$

$S=E(G)-P_{i} \cup Q_{i} \cup R_{i}$

is a simple graphoidal cover of $\mathrm{G}$ in which $w_{11}$ is not internal. Therefore $\eta_{s}(G) \leq$ $q-p+1$. Now let $\psi$ be any graphoidal cover of $\mathrm{G}$. If $\psi$ contains only paths, then atleast four vertices can not be made internal. If $\psi$ contains atleast one cycle, then atleast one vertex can not be made internal. Thereforet $\psi \geq 1$ Hence $\eta_{s}(G) \geq q-p+1$. Thus $\eta_{s}(G)=q-p+1$.

Case (ii) When $\mathrm{m}=$ odd and $\mathrm{n}=$ even, The collection of paths of $\mathrm{G}$ are

$P_{i}=\left(w_{1 j}, w_{1(j+1)}, w_{2 j}, w_{1 j}\right): 1 \leq j \leq(n-1)$

$Q_{i}=\left(w_{i j}, w_{(i+1) j}, w_{(i+1)(j+1)}, w_{i j}\right): i=2,3 \ldots(m-1) ; j=1,3,5 \ldots(n-1)$

$R=\left(w_{1 n}, w_{2 n}, w_{3 n}\right)$

$S=E(G)-P_{i} \cup Q_{i} \cup R$

is a simple graphoidal cover of $\mathrm{G}$ in which $w_{11}$ is not internal. Therefore $\eta_{s}(G) \leq$ $q-p+1$. Now let $\psi$ be any graphoidal cover of $\mathrm{G}$. If $\psi$ contains only paths, then atleast four vertices can not be made internal. If $\psi$ contains atleast one cycle, then atleast one vertex can not be made internal. Therefore $t_{\psi} \geq 1$. Hence $\eta_{s}(G) \geq q-p+1$. Thus $\eta_{s}(G)=q-p+1$.

Case (iii) When $\mathrm{m}=$ even and $\mathrm{n}=$ odd, The collection of paths of $\mathrm{G}$ are

$$
\begin{aligned}
& P_{i}=\left(w_{1 j}, w_{1(j+1)}, w_{2 j}, w_{1 j}\right) ; 1 \leq j \leq(n-1) \\
& Q_{i}=\left(w_{i j}, w_{(i+1) j}, w_{(i+1)(j+1)}, w_{i j}\right): 2 \leq j \leq(m-1) ; j=1,3,5 \ldots(n-2) \\
& R_{i}=\left(w_{i(n-1)}, w_{i n}, w_{(i+1) n}, w_{i(n-1)}\right): i=3,5 . .(m-1)
\end{aligned}
$$


$S=\left(w_{1 n}, w_{2 n}, w_{3 n}\right)$

$T=E(G)-P_{i} \cup Q_{i} \cup R_{i} \cup S$

is a simple graphoidal cover of $\mathrm{G}$ in which $w_{11}$ is not internal. Therefore $\eta_{s}(G) \leq$ $q-p+1$. Now let $\psi$ be any graphoidal cover of $\mathrm{G}$. If $\psi$ contains only paths, then atleast four vertices can not be made internal. If $\psi$ contains atleast one cycle, then atleast one vertex can not be made internal. Thereforet $\psi \geq 1$. Hence $\eta_{s}(G) \geq q-p+1$. Thus $\eta_{s}(G)=q-p+1$.

Case (iv) When $\mathrm{m}=\mathrm{n}=$ even, The collection of paths of $\mathrm{G}$ are

$P_{i}=\left(w_{1 j}, w_{1(j+1)}, w_{2 j}, w_{1 j}\right): 1 \leq j \leq(n-1)$

$Q_{i}=\left(w_{i j}, w_{(i+1) j}, w_{(i+1)(j+1)}, w_{i j}\right): 2 \leq j \leq(m-1) ; j=1,3,5 \ldots(n-1)$

$R=\left(w_{1 n}, w_{2 n}, w_{3 n}\right)$

$S=E(G)-P_{i} \cup Q_{i} \cup R$

is a simple graphoidal cover of $\mathrm{G}$ in which $w_{11}$ is not internal. Therefore $\eta_{s}(G) \leq$ $q-p+1$. Now let $\psi$ be any graphoidal cover of G. If $\psi$ contains only paths, then atleast four vertices can not be made internal. If $\psi$ contains atleast one cycle, then atleast one vertex can not be made internal. Thereforet $\psi \geq 1$. Hence $\eta_{s}(G) \geq q-p+1$. Thus $\eta_{s}(G)=q-p+1$.

Theorem 14. For $C_{m} \otimes C_{n}, m, n \geq 3$ then

$$
\eta_{s}(G)= \begin{cases}q-p+1 & \text { if } m=n=3 \\ q-p & \text { if } m \geq 3 \text { and } n \geq 4\end{cases}
$$

Proof. Let Edge Set $\mathrm{E}(\mathrm{G})=\left\{w_{i j} w_{i(j+1)}: 1 \leq i \leq m ; 1 \leq j \leq(n-1)\right\}$ $\cup\left\{w_{i n} w_{i 1}: 1 \leq i \leq m\right\} \cup\left\{w_{i n} w_{(i+1) 1}: 1 \leq i \leq(m-1)\right\} \cup\left\{w_{i 1} w_{(i+1) n}: 1 \leq\right.$ $i \leq(m-1)\} \cup\left\{w_{i j} w_{(i+1) j}: 1 \leq i \leq(m-1) ; 1 \leq j \leq n\right\} \cup\left\{w_{m j} w_{1 j}: 1 \leq\right.$ $j \leq n\} \cup\left\{w_{1 j} w_{m(j+1)}: 1 \leq j \leq(n-1)\right\} \cup\left\{w_{m j} w_{1(j+1)}: 1 \leq i \leq(m-1)\right\} \cup$ $\left\{w_{i j} w_{(i+1)(j+1)}: 1 \leq i \leq(m-1) ; 1 \leq j \leq(n-1)\right\} \cup\left\{w_{i j} w_{(i+1)(j-1)}: 1 \leq i \leq\right.$ $(m-1) ; 2 \leq j \leq n\} \cup\left\{w_{11} w_{m n}\right\} \cup\left\{w_{1 n} w_{m 1}\right\}$

When $\mathrm{m}=\mathrm{n}=3$, The collection of paths of $\mathrm{G}$ are

$P_{i}=\left(w_{i 1}, w_{i 2}, w_{i 3}, w_{i 1}\right): i=1,2,3$

$Q=\left(w_{11}, w_{21}, w_{31}, w_{11}\right)$

$R=E(G)-P_{i} \cup Q$

is a simple graphoidal cover of $\mathrm{G}$ in which $w_{11}$ is the only vertex which is not internal. Therefore $\eta_{s}(G) \leq q-p+1$. Now let $\psi$ be any graphoidal cover of $\mathrm{G}$. If 
$\psi$ contains only paths, then no vertex can be made internal, since every path is an edge. If $\psi$ contains atleast one cycle, then atleast one vertex can not be made internal. Therefore $t_{\psi} \geq 1$. Hence $\eta_{s}(G) \geq q-p+1$. Thus $\eta_{s}(G)=q-p+1$.

when $\mathrm{m} \geq 3$ and $n \geq 4$, The collection of paths of $\mathrm{G}$ are

$P_{i}=\left(w_{i 1}, w_{i 2}, w_{i 3}, \ldots . w_{i n}, w_{i 1}\right): i=1,2,3 \ldots m$

$Q=\left(w_{11}, w_{21}, w_{31}, w_{41} \ldots . . w_{m 1}, w_{11}\right)$

$R=\left(w_{22}, w_{11}, w_{m n}\right)$

$S=E(G)-P_{i} \cup Q \cup R$

is a simple graphoidal cover of $\mathrm{G}$ in which all the vertices made internal. By corollary $(5), \eta_{s}(G)=q-p$.

Theorem 15. For $P_{m} \otimes C_{n}, m \geq 2, n \geq 3$ then $\eta_{s}(G)=q-p+2$.

Proof. Let Edge Set $\mathrm{E}(\mathrm{G})=\left\{w_{i j} w_{i(j+1)}: 1 \leq i \leq m ; 1 \leq j \leq(n-1)\right\}$ $\cup\left\{w_{i n} w_{i 1}: 1 \leq i \leq m\right\} \cup\left\{w_{i n} w_{(i+1) 1}: 1 \leq i \leq(m-1)\right\} \cup\left\{w_{i 1} w_{(i+1) n}: 1 \leq i \leq\right.$ $(m-1)\} \cup\left\{w_{i j} w_{(i+1) j}: 1 \leq i \leq(m-1), 1 \leq j \leq n\right\} \cup\left\{w_{i j} w_{(i+1)(j+1)}: 1 \leq i \leq\right.$ $(m-1), 1 \leq j \leq(n-1)\} \cup\left\{w_{i j} w_{(i+1)(j-1)}: 1 \leq i \leq m-1,2 \leq j \leq n\right\}$. Here We consider two cases

Case(i) When $\mathrm{m}=2$ and $\mathrm{n} \geq 3$, The collection of path of $\mathrm{G}$ are

$P_{i}=\left(w_{i 1}, w_{i 2}, w_{i 3}, \ldots . w_{i n}, w_{i 1}\right): i=1,2,3 \ldots m$

$Q=E(G)-P_{i}$

is a simple graphoidal cover of $\mathrm{G}$ in which $w_{11}$ and $w_{21}$ are not an internal. Therefore $\eta_{s}(G) \leq q-p+2$. Now let $\psi$ be any graphoidal cover of G. Here we have two sub cases.

Sub case(i) When $\mathrm{m}=2$ and $\mathrm{n}=3$, If $\psi$ contains only paths, no vertices can not be made internal, since each path is an edge. If $\psi$ contains atleast one cycle, two vertices can not be made internal, since each path is an edge. Thereforet $\psi 2$. Hence $\eta_{s}(G) \geq q-p+2$. Thus $\eta_{s}(G)=q-p+2$.

Sub case (ii) When $\mathrm{m}=2, \mathrm{n}>3$, If $\psi$ contains only paths, four vertices can not be made internal. If $\psi$ contains atleast one cycle, two vertices can not be made internal. Thereforet $\psi \geq 2$. Hence $\eta_{s}(G) \geq q-p+2$. Thus $\eta_{s}(G)=q-p+2$.

Case (ii) When $m \geq 3$ and $n \geq 3$, The collection of path of $G$ are $P_{i}=\left(w_{i 1}, w_{i 2}, w_{i 3}, \ldots . w_{i n}, w_{i 1}\right): i=1,2,3 \ldots m$ 
$Q=\left(w_{11}, w_{21}, w_{31}, w_{11} \ldots . . w_{m 1}\right)$

$R=E(G)-P_{i} \cup Q$

is a simple graphoidal cover of $\mathrm{G}$ in which $w_{11}$ and $w_{m 1}$ are not an internal. Therefore $\eta_{s}(G) \leq q-p+2$. Now let $\psi$ be any graphoidal cover of G. If $\psi$ contains only paths, then atleast six vertices can not be made internal. If $\psi$ contains atleast one cycle,then atleast two vertex can not be made internal. Thereforet $\psi \geq 2$. Hence $\eta_{s}(G) \geq q-p+2$. Thus $\eta_{s}(G)=q-p+2$.

\section{References}

[1] B.D. Acharya and E. Sampath Kumar, Graphoidal covers and graphoidal covering number of a graph, Indian Journal Pure Appl. Math., 18, No. 10 (1987), 882-890.

[2] S. Arumugam, B.D. Acharya and E. Sampath Kumar, Graphoidal covers of a graph: A creative review, In: Proceedings of National Workshop on Graph Theory and Applications, Manaonmaniam Sundaranar University, Tirunelveli, Tata Mcgrew Hill, New Delhi (1997), $1-28$.

[3] S. Arumuagm and J. Suresh Sussela, Acyclic graphoidal covers and path partitions in a graph, Discrete Mathematics, 190 (1998), 66-77, doi: 10.1016/S0012-365X(98)00032-6.

[4] S. Arumugam and I. Sahul Hamid, Simple graphoidal covers in a graph, J. Combin. Math. Combin. Comput., 64 (2008), 79-95.

[5] S. Arumugam and C. Pakkiam, Graphs with unique minimum graphoidal cover, Indian J. Pure Appl. Math., 25 (1994), 1147-1153.

[6] S. Arumugam and C. Pakkiam, Graphoidal bipartite graphs, Graphs and Coimbinatorics, 10 (1994), 305-310, doi: 10.1007/BF02986680.

[7] S. Arumugam and C. Pakkiam, The graphoidal covering number of directed graphs, J. Math. Phy. Sci., 29 (1995), 33-41.

[8] C. Pakkiam and S. Arumugam, On the graphoidal covering number of a graph, Indian J. Pure Appl. Math., 20 (1989), 330-333.

[9] C. Pakkiam, S. Arumugam, The graphoidal coveting number of unicyclic graphs, Indian J. Pure Appl. Math., 23 (1992), 141-143.

[10] F. Harary, Graph Theory, Addison-Wesley, Reading, MA, 1969.

[11] R. Hammack, W. Imrich, S. Klavžar, Handbook of Product Graphs, CRC Press, New York, USA, 2011. 